\title{
ASYMPTOTIC ANALYSIS OF THE ONE-DIMENSIONAL GINZBURG-LANDAU EQUATIONS NEAR SELF-DUALITY
}

\author{
BY \\ Y. ALMOG \\ Department of Applied Mathematics and Computer Science, The Weizmann Institute of Science, \\ Rehovot, Israel
}

\begin{abstract}
It is known that when the Ginzburg-Landau parameter $\kappa=1 / \sqrt{2}$ the onedimensional Ginzburg-Landau equations exhibit self-duality and may be reduced into a pair of first-order ODE. The present asymptotic analysis initially focuses on infinite samples of superconductors for which $|\kappa-1 / \sqrt{2}| \ll 1$. It is shown that when the value of the applied magnetic field at infinity lies between $\kappa$ and $1 / \sqrt{2}$ a superconducting solution exists. It is later shown, for arbitrary values of $\kappa$, that no solution, other than the normal state, can exist for applied magnetic field values that lie outside the above interval.
\end{abstract}

1. Introduction. Solutions for the one-dimensional Ginzburg-Landau equations, which model superconductivity, have been discussed mostly from the point of view of linear bifurcation theory. Millman and Keller [12] provided the framework for obtaining the applied magnetic field for which the bifurcation from the normal state occurs. As an example, they have discussed the one-dimensional case on a finite interval. Chapman [5] provided several other examples, among them the one-dimensional case on an infinite sample.

We shall subsequently refer to superconducting materials for which the GinzburgLandau parameter $\kappa<1 / \sqrt{2}$ as type I superconductors and to those superconductors for which $\kappa>1 / \sqrt{2}$ as type II. Chapman [5] demonstrated for the above-mentioned example that when the applied magnetic field $h$ is equal to $\kappa$ a superconducting branch bifurcates from the normal state solution. This superconducting solution, for the linearized Ginzburg-Landau equations, can exist only for $h<\kappa(h>\kappa)$ for type II (type I) superconductors. Chapman also showed, in a follow-up contribution [6], that the superconducting solution is stable (unstable) for type I (type II) superconductors, demonstrating that the value of what has been termed the upper critical field (at which the solution ceases to be normal when the applied magnetic field is gradually decreased) is exactly $\kappa$.

Linear bifurcation theory is an effective tool, when seeking the value of the parameter at which bifurcation occurs and the solutions themselves near the bifurcation. It cannot,

Received January 9, 1997.

1991 Mathematics Subject Classification. Primary 82D55, 58F14. 
however, predict highly nonlinear effects. For instance, we cannot be sure that no solution other than the normal one exists when the applied field is larger than the upper critical field (for type II superconductors). Furthermore, there is no guarantee that any solution, other than the normal state, would still exist, if we decrease the applied magnetic field further to values that are not close to the upper critical field.

In order to properly address the questions above, which all involve nonlinear effects, it is necessary to discuss solutions of the nonlinear Ginzburg-Landau equations (in contrast to linear bifurcation theory which investigates solutions of the linearized system). Such a family of solutions can be obtained when the Ginzburg-Landau equations exhibit what has been termed self-duality [1], [2]. These solutions can be obtained, however, only for a specific value of $\kappa$, the Ginzburg-Landau parameter (i.e., $\kappa=1 / \sqrt{2}$ ). In the present work we look first at superconductors with $\kappa$ close to $1 / \sqrt{2}$. We shall be able to obtain in this way the range of applied magnetic field values for which superconducting solutions exist. Motivated by the results of this asymptotic analysis, we shall be able to prove a nonexistence theorem valid for arbitrary values of $\kappa$.

Consider a superconducting material given in an infinite domain. The magnetic field $\mathbf{H}$ is taken to be directed along the $z$ axis and the magnetic vector potential $\mathbf{A}$ to be directed along the $y$ axis. We assume that all functions depend solely on $x$, hence, $H_{3}=\frac{d A_{2}}{d x}$, or simply, $H=\frac{d A_{2}}{d x}=A_{2}^{\prime}$. (Since $A_{2}$ is the only nonzero component of $\mathbf{A}$ we shall subsequently denote it by $\mathbf{A}$.) The Ginzburg-Landau equations may then be written in their one-dimensional form [5]:

$$
\begin{aligned}
& \frac{\psi^{\prime \prime}}{\kappa^{2}}=\psi^{3}-\psi+A^{2} \psi, \\
& A^{\prime \prime}=\psi^{2} A,
\end{aligned}
$$

where $\psi$ is the superconducting order parameter (the gauge has been chosen so that $\psi$ would be real). The boundary conditions satisfied by $\psi$ and $A$ are

$$
\begin{aligned}
& \psi^{\prime} \rightarrow 0 \text { as }|x| \rightarrow \infty \text {, } \\
& A^{\prime} \rightarrow h \text { as }|x| \rightarrow \infty \text {, }
\end{aligned}
$$

where $h$ is the applied magnetic field at infinity. Note that at least one solution for (1.1) always exists, namely

$$
\psi=0 ; \quad A=h x+c .
$$

We shall refer to (1.2) as the normal solution. Any other solution of (1.1) would be called a superconducting solution. We shall be interested only in solutions for which $\psi \geq 0$.

When $\kappa=1 / \sqrt{2}$ (1.1) exhibit what has been termed self-duality, so that for $h=1 / \sqrt{2}$, solutions of (1.1) are also solutions of the following pair of first-order ODEs:

$$
\begin{aligned}
& \sqrt{2} A^{\prime}=1-\psi^{2}, \\
& \sqrt{2} \psi^{\prime}=-\psi A .
\end{aligned}
$$


[There may be, of course, different solutions for (1.1).] A family of solutions can be found for (1.3)

$$
x=\int_{\psi^{2}(0)}^{\psi^{2}} \frac{-d\left(\psi^{2}\right)}{\sqrt{2} \psi^{2}\left(\psi^{2}-c-\log \psi^{2}\right)^{1 / 2}}
$$

where $c$ may be any number greater than 1 . Chapman [5] plots $\psi$ and $A^{\prime}$ versus $x$ for various values of $c$. It is easy to show that the value of $\psi^{2}(0)$ increases as $c \rightarrow 1$. For $c=1$ (1.4) does not satisfy anymore (1.1c) and (1.1d) but instead satisfies different boundary conditions for $x \rightarrow \infty$ and $x \rightarrow-\infty$, i.e., while the boundary condition at $x \rightarrow \infty$ remains unaltered, we must have $\psi=1, A=0$ as $x \rightarrow-\infty$.

In the next section we provide an asymptotic approximation for superconducting solutions of (1.1) for $0<|\kappa-1 / \sqrt{2}| \ll 1$. Motivated by the results of Sec. 2 we prove in Sec. 3 that superconducting solutions of (1.1) cannot exist when the applied magnetic field $h$ does not lie between $\kappa$ and $1 / \sqrt{2}$. In the last section we examine the significance of our results to time-dependent analyses.

2. Asymptotic solutions near self-duality. We first define a new parameter

$$
\varepsilon=\frac{1}{\kappa^{2}}-2
$$

and rewrite (1.1) in the form

$$
\begin{aligned}
(2+\varepsilon) \psi^{\prime \prime} & =\psi^{3}-\psi+\psi A^{2}, \\
A^{\prime \prime} & =\psi^{2} A, \\
\psi^{\prime} & \stackrel{|x| \rightarrow \infty}{\longrightarrow} 0, \\
A^{\prime} & \stackrel{\longrightarrow}{|x| \rightarrow \infty} h=\frac{1}{\sqrt{2}}+h_{1} \varepsilon .
\end{aligned}
$$

Our goal is to approximate the solution of the above problem for $\varepsilon \ll 1$. Since for $\varepsilon=0$ the solution is known [and is given by (1.4)] we introduce the expansion

$$
\begin{aligned}
& \psi \cong \psi_{0}+\varepsilon \psi_{1}+\varepsilon^{2} \psi_{2}+O\left(\varepsilon^{3}\right), \\
& A \cong A_{0}+\varepsilon A_{1}+\varepsilon^{2} A_{2}+O\left(\varepsilon^{3}\right) .
\end{aligned}
$$

As was pointed out in the preceding section, we have a family of solutions for the case $\varepsilon=0$. We expect, however, in view of the results of linear bifurcation theory [5], to have a unique superconducting solution for $\varepsilon \neq 0$. Thus, we expect that the $O(\varepsilon)$ balance would be solvable only for a specific $\psi_{0}$, or equivalently, in view of (1.4), for a specific value of $c$. The $O(\varepsilon)$ balance

$$
\begin{aligned}
2 \psi_{1}^{\prime \prime} & =\left(3 \psi_{0}^{2}-1+A_{0}^{2}\right) \psi_{1}+2 \psi_{0} A_{0} A_{1}-\psi_{0}^{\prime \prime}, \\
A_{1}^{\prime \prime} & =\psi_{0}^{2} A_{1}+2 \psi_{0} A_{0} \psi_{1}, \\
\psi^{\prime} & \underset{|x| \rightarrow \infty}{\longrightarrow} 0, \\
A^{\prime} & \stackrel{|x| \rightarrow \infty}{\longrightarrow} h_{1}
\end{aligned}
$$


is a fourth-order linear system with nonconstant coefficients. In order to solve (2.4) we introduce the following pair of functions:

$$
\begin{aligned}
& f=A^{\prime}-\frac{1}{\sqrt{2}}\left(1-\psi^{2}\right), \\
& g=\sqrt{2} \psi^{\prime}+\psi A .
\end{aligned}
$$

Using the above definitions we can rewrite $(2.2 \mathrm{a})$ and $(2.2 \mathrm{~b})$ in the alternative form

$$
\begin{gathered}
\sqrt{2} g^{\prime}-\sqrt{2} f \psi-A g=-\varepsilon \psi^{\prime \prime}, \\
f^{\prime}-\psi g=0 .
\end{gathered}
$$

Upon expanding $f$ and $g$ into power series of $\varepsilon$,

$$
\begin{aligned}
& f=f_{0}+\varepsilon f_{1}+\varepsilon^{2} f_{2}+O\left(\varepsilon^{3}\right), \\
& f=g_{0}+\varepsilon g_{1}+\varepsilon^{2} g_{2}+O\left(\varepsilon^{3}\right)
\end{aligned}
$$

and noticing that, as a result of (1.3), $f_{0}$ and $g_{0}$ must identically vanish, we obtain the following fourth-order system which is equivalent to (2.4):

$$
\begin{gathered}
A_{1}^{\prime}+\sqrt{2} \psi_{0} \psi_{1}=f_{1}, \\
\sqrt{2} \psi_{1}^{\prime}+\psi_{1} A_{0}+\psi_{0} A_{1}=g_{1}, \\
\sqrt{2} g_{1}^{\prime}-\sqrt{2} f_{1} \psi_{0}-A_{0} g_{1}=-\psi_{0}^{\prime \prime}, \\
f_{1}^{\prime}-\psi_{0} g_{1}=0 .
\end{gathered}
$$

In view of (2.8a) and the asymptotic behavior for large $x$ of $\psi_{0}$ [utilizing (1.4) it is easy to show that $\left.\psi_{0} \sim O\left(e^{-x^{2} / 4}\right)\right], f_{1}$ must satisfy the following boundary value problem:

$$
\begin{aligned}
& f_{1}^{\prime \prime}-\psi_{0}^{2} f_{1}=\frac{1}{\sqrt{2}} \psi_{0} \psi_{0}^{\prime \prime}, \\
& f_{1} \underset{|x| \rightarrow \infty}{\longrightarrow} h_{1} .
\end{aligned}
$$

Integrating the product of $(2.9 \mathrm{a})$ with $A_{0}$ between $x$ and $\infty(x>0)$ we obtain

$$
\left(\frac{f_{1}}{A_{0}}\right)^{\prime}=\frac{1}{4} \psi_{0}^{2}-\frac{h_{1}}{\sqrt{2} A_{0}^{2}}
$$

Hence, for $x>0, f_{1}$ is expressible in the form

$$
f_{1}=-\frac{1}{4} A_{0} \int_{x}^{\infty} \psi_{0}^{2} d x+\frac{h_{1}}{\sqrt{2}} A_{0} \int_{x}^{\infty} \frac{1}{A_{0}^{2}} d x .
$$

For $x<0$ we simply have $f_{1}(x)=f_{1}(-x)$.

Clearly, $f_{1}$ is continuous at $x=0$. However, to be a valid solution of (2.9) $f_{1}^{\prime}$ needs to be continuous at $x=0$ as well. Equivalently, since $f_{1}$ is even, we may require $f_{1}^{\prime}=0$. This requirement leads to the following solvability condition:

$$
h_{1}=-\frac{\sqrt{2}}{4} \frac{\int_{0}^{\infty} \psi_{0}^{2} d x}{\int_{0}^{\infty} \frac{\psi_{0}^{2}}{\left(A_{0}^{\prime}\right)^{2}} d x} .
$$

The right-hand side of (2.12) is a function of $c$, or alternatively of $\psi_{0}(0)=\bar{\psi}$. As will be subsequently demonstrated [cf. (2.22)], if (2.12) holds, solutions for (2.4) can be found. 
Hence, (2.12) serves as both a necessary and sufficient condition for the solvability of (2.4).

When $\bar{\psi} \ll 1$ the approximations

$$
\begin{aligned}
& \psi_{0} \cong \bar{\psi} e^{-x^{2} / 4}+O\left(\bar{\psi}^{2}\right), \\
& A_{0}^{\prime} \cong \frac{1}{\sqrt{2}}+O(\bar{\psi})
\end{aligned}
$$

may be substituted into (2.12) to obtain

$$
h_{1} \cong-\frac{1}{4 \sqrt{2}}+O\left(\bar{\psi}^{2}\right) .
$$

Since by $(2.1) \kappa \cong \frac{1}{\sqrt{2}}-4 \sqrt{2} \varepsilon+O\left(\varepsilon^{2}\right)$, it follows that

$$
h \cong \kappa+O\left(\bar{\psi}^{2}, \varepsilon^{2}\right)
$$

which is in accordance with the results of linear bifurcation theory [5].

By (1.3a) and (1.4) $A_{0}^{\prime}(x, \bar{\psi})$ is a decreasing function of $\bar{\psi}$. Therefore, $h_{1}$ must be an increasing function of $\bar{\psi}$. We conclude that a solution to (2.4) can exist only when

$$
-\frac{1}{4 \sqrt{2}}<h_{1}<0
$$

For type I (type II) superconductors we may express the above condition in the alternative form $\kappa<h<1 / \sqrt{2}(1 / \sqrt{2}<h<\kappa)$. While the lower bound for $h_{1}$ is expected in view of linear bifurcation theory results [5], the upper bound cannot be detected by any linear theory since it involves highly nonlinear effects. It is, however, intuitively expected since for $h=1 / \sqrt{2}$ the free energies of the superconducting state and the normal state are equal.

Figure 1 displays the dependence of $\bar{\psi}$ on $-4 \sqrt{2} h_{1}$ according to (2.12). It can be seen that as $h_{1} \rightarrow-\frac{1}{4 \sqrt{2}}, \bar{\psi} \rightarrow 0$ and that as $h_{1} \rightarrow 0, \bar{\psi} \rightarrow 1$. A monotonic dependence of $\bar{\psi}$ on $h_{1}$ is observed.

Once the derivation of the relation (2.12) between the applied magnetic field and the leading-order solution is complete, the energy of the solutions may be approximated. The Ginzburg-Landau equations (1.1) are the Euler-Lagrange equations associated with the energy functional

$$
E=\int_{-\infty}^{\infty}\left[f^{2}+g^{2}+(\sqrt{2}-2 h) A^{\prime}+\left(h^{2}-\frac{1}{2}\right)+\varepsilon\left(\psi^{\prime}\right)^{2}\right] d x
$$

reflecting the difference between the energy of $(\psi, A)$ and the normal state (1.2). For the critical case $\kappa=1 / \sqrt{2}, h=1 / \sqrt{2}$ the energy vanishes identically. For nearly critical cases it is of $O(\varepsilon)$.

Introducing the expansion

$$
E \cong \varepsilon E_{1}+O\left(\varepsilon^{2}\right)
$$

together with (2.3) and (2.7) we obtain

$$
E_{1}=\int_{-\infty}^{\infty}\left[\sqrt{2} h_{1} \psi_{0}^{2}+\left(\psi_{0}^{\prime}\right)^{2}\right] d x
$$




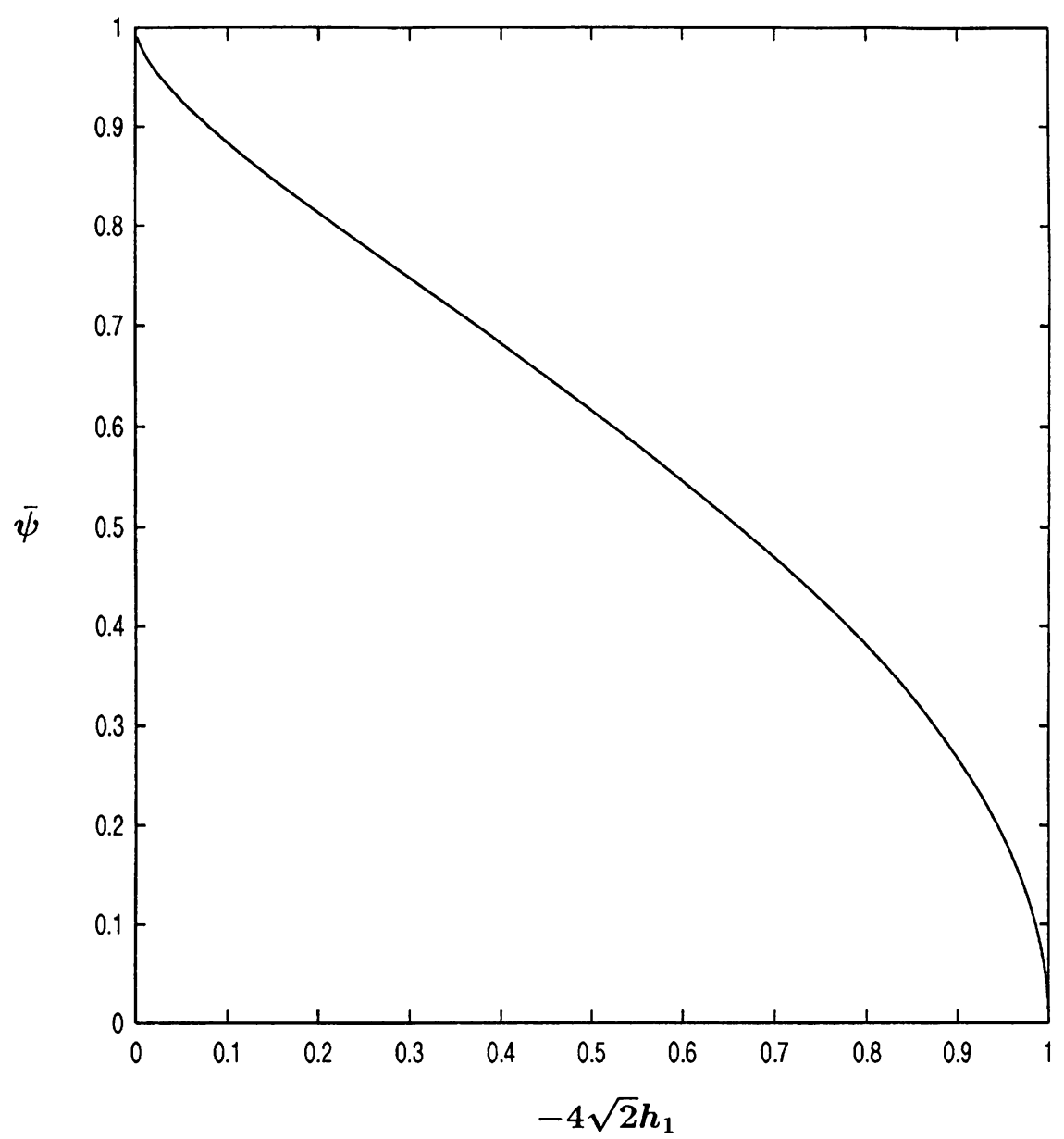

FIG. 1. Variation of $\bar{\psi}$, the maximal superconducting order parameter, with $-4 \sqrt{2} h_{1}$, representing the applied magnetic field

Integration by parts then yields

$$
E_{1}=\int_{-\infty}^{\infty}\left[\sqrt{2} h_{1} \psi_{0}^{2}+\frac{1}{4} \psi_{0}^{2}\left(1-\psi_{0}^{2}\right)\right] d x
$$

We shall first demonstrate that $E_{1}>0$ independently of $h_{1}$ 's value. Define

$$
I_{n}=\int_{-\infty}^{\infty}\left(\psi_{0}^{2}\right)^{n} d x
$$

Then,

$$
\sqrt{2} h_{1}=-\frac{1}{4} \frac{I_{1}}{\int_{-\infty}^{\infty} \psi_{0}^{2}\left(1-\psi_{0}^{2}\right)^{-2} d x}>-\frac{1}{4} \frac{I_{1}}{\sum_{n=1}^{\infty} I_{n}}
$$

and hence by (2.18), we obtain

$$
4 E_{1}>\frac{\sum_{n=1}^{\infty}\left(I_{1} I_{n+1}-I_{2} I_{n}\right)}{\sum_{n=1}^{\infty} I_{n}}
$$


Application of Hölder's inequality (with $p=n /(n-1), q=n$ ) yields

$$
I_{2} \leq I_{1}^{\frac{n-1}{n}} I_{n+1}^{1 / n} ; \quad I_{n} \leq I_{n+1}^{\frac{n-1}{n}} I_{1}^{1 / n} .
$$

Hence, $I_{2} I_{n} \leq I_{1} I_{n+1}$ from which positivity of $E_{1}$ follows.

Recalling that $\varepsilon$ is positive for type I superconductors and negative for type II, and that the energy of the normal state vanishes, we conclude that $\left(\psi_{0}, A_{0}\right)$ can serve as a global minimizer for $E$ only for type II superconductors. This is, however, a highly expected result since, in general, superconducting solutions were found to be stable only when $\kappa>1 / \sqrt{2}[6]$.

Dorsey [9] approximated the energy of the solution for the asymptotic problem, when $\psi \rightarrow 1$ and $A \rightarrow 0$ as $x \rightarrow-\infty$, near self-duality. The energy of such a solution has been termed "the surface tension" since it presents the surface energy of a nor$\mathrm{mal} /$ superconducting interface. We shall now demonstrate the relation between $E_{1}$ and Dorsey's [9] approximation.

We first derive an upper bound for $\psi_{0}$. Observing that $A_{0}>A_{0}^{\prime}(0) x$ for $x>0$ we substitute in $(1.3 \mathrm{~b})$ to derive the following crude estimate:

$$
\psi_{0} \leq \exp \left\{-\frac{1}{4}\left[1-\psi_{0}^{2}(0)\right] x^{2}\right\},
$$

from which we find

$$
\int_{0}^{\infty} \psi_{0}^{2} d x \leq \frac{1}{2}\left[\frac{2 \pi}{1-\psi_{0}^{2}(0)}\right]^{1 / 2} .
$$

Next, it is easy to show that on some finite interval $0 \leq x \leq l$, for sufficiently small values of $1-\psi_{0}(0)$, we have

$$
\frac{\psi_{0}^{2}}{\left(1-\psi_{0}^{2}\right)^{2}} \geq \frac{K}{\left[1-\psi_{0}^{2}(0)\right]^{2}}
$$

where $K$ is independent of $\psi_{0}(0)$. Hence,

$$
\int_{0}^{\infty} \frac{\psi_{0}^{2}}{\left(1-\psi_{0}^{2}\right)^{2}} d x \geq \frac{K l}{\left[1-\psi_{0}^{2}(0)\right]^{2}} .
$$

Combining (2.12), (2.17), (2.19), and (2.20) we then have

$$
\lim _{h \rightarrow 0} E_{1}=\int_{-\infty}^{\infty}\left(\psi_{0}^{\prime}\right)^{2} d x .
$$

Comparison with [9] reveals that $E_{1} \varepsilon$ tends, as $h_{1} \rightarrow 0$, to be twice the approximated value, near self-duality, of the surface tension. The numerical calculation in [9] then finds

$$
\lim _{h_{1} \rightarrow 0} E_{1}=0.388 \text {. }
$$

We conclude this section by obtaining the next order term, demonstrating that our asymptotic scheme works for higher-order terms as well. To this end we first obtain the general solution of $(2.4)$

$$
\left[\begin{array}{l}
A_{1} \\
\psi_{1}
\end{array}\right]=C_{1}\left[\begin{array}{l}
A_{0}^{\prime} \\
\psi_{0}^{\prime}
\end{array}\right]+C_{2}\left[\begin{array}{l}
v A_{0}^{\prime} \\
\psi_{1 h}
\end{array}\right]+\left[\begin{array}{l}
A_{1 p} \\
\psi_{1 p}
\end{array}\right]
$$


The first term on the right-hand side appears very often when applying perturbation expansions (cf. [11]). The functions $v$ and $\psi_{1 h}$ appearing in the second term are defined as follows:

$$
\begin{aligned}
v & =\int_{0}^{x} \frac{\psi_{0}^{2}}{\left(A_{0}^{\prime}\right)^{2}} d x \\
\psi_{1 h} & =-\left[\frac{\psi_{0}}{\sqrt{2} A_{0}^{\prime}}-v \psi_{0}^{\prime}\right] .
\end{aligned}
$$

The last term on the right-hand side of (2.22) is a particular solution of (2.4) and is expressible in the form

$$
\begin{aligned}
& A_{1 p}=\left(u_{1}+u_{2} v\right) A_{0}^{\prime} \\
& \psi_{1 p}=\frac{1}{\sqrt{2} \psi_{0}}\left(f_{1}-A_{1 p}^{\prime}\right)
\end{aligned}
$$

in which

$$
\begin{aligned}
& u_{1}=-\int_{0}^{x} \frac{\sqrt{2} A_{0} A_{0}^{\prime} f_{1}}{\psi_{0}^{2}} d x, \\
& u_{2}=\int_{0}^{x} \frac{\sqrt{2} v A_{0} A_{0}^{\prime} f_{1}}{\psi_{0}^{2}} d x .
\end{aligned}
$$

In the same manner in which we have obtained (2.9) we obtain the following boundary value problem for $f_{2}$ :

$$
\begin{gathered}
f_{2}^{\prime \prime}-\psi_{0}^{2} f_{2}=\left(\psi_{1} g_{1}\right)^{\prime}+\frac{1}{\sqrt{2}} \psi_{0}\left[\sqrt{2} f_{1} \psi_{1}+A_{1} g_{1}-\psi_{1}^{\prime \prime}\right] \\
f_{2} \underset{|x| \rightarrow \infty}{\stackrel{\longrightarrow}{\longrightarrow}} 0 .
\end{gathered}
$$

Note that, unlike $f_{1}, f_{2}$ is not necessarily even. We therefore obtain two solvability conditions: The first one ensures continuity of $f_{2}$ at $x=0$,

$$
\int_{-\infty}^{\infty}\left[A_{0}\left(\psi_{1} g_{1}\right)^{\prime}-\psi_{0}^{\prime}\left(\sqrt{2} f_{1} \psi_{1}+A_{1} g_{1}-\psi_{1}^{\prime \prime}\right)\right] d x=0
$$

and the second condition guarantees the continuity of $f_{2}^{\prime}$ at $x=0$,

$$
\begin{gathered}
\int_{0}^{\infty} \frac{\psi_{0}^{2}}{\left(A_{0}^{\prime}\right)^{2}}\left\{\int_{x^{\prime}}^{\infty}\left[A_{0}\left(\psi_{1} g_{1}\right)^{\prime}-\psi_{0}^{\prime}\left(\sqrt{2} f_{1} \psi_{1}+A_{1} g_{1}-\psi_{1}^{\prime \prime}\right)\right]_{o} d x^{\prime \prime}\right\} d x^{\prime} \\
+\int_{0}^{\infty} \frac{1}{A_{0}^{\prime}}\left[\left(\psi_{1} g_{1}\right)^{\prime}+\frac{1}{\sqrt{2}} \psi_{0}\left(\sqrt{2} f_{1} \psi_{1}+A_{1} g_{1}-\psi_{1}^{\prime \prime}\right)\right]_{e} d x^{\prime}=0
\end{gathered}
$$

wherein

$$
\begin{aligned}
& {[u]_{e}(x)=\frac{1}{2}[u(x)+u(-x)],} \\
& {[u]_{e}(x)=\frac{1}{2}[u(x)-u(-x)]}
\end{aligned}
$$

are, respectively, the even and the odd part of the function $u$.

Substituting (2.22) into (2.26) we note that the contribution of the second and the third terms on the right-hand side of $(2.22)$, which are both even, to the left-hand side 
of (2.26) vanishes. We are therefore left with the condition

$$
C_{1} \int_{-\infty}^{\infty}\left[A_{0}\left(\psi_{0}^{\prime} g_{1}\right)^{\prime}-\psi_{0}^{\prime}\left(\sqrt{2} f_{1} \psi_{0}^{\prime}+A_{0}^{\prime} g_{1}-\psi_{0}^{(3)}\right)\right] d x=0 .
$$

It is not difficult to show that the above condition is satisfied for arbitrary $C_{1}$. By invoking symmetry conditions once again, it is readily seen that $(2.27)$ is satisfied for arbitrary $C_{1}$ as well. Hence, $C_{1}$ remains undetermined. This indeterminacy is highly expected in view of the translational invariance of $(2.2)$, i.e., if $\psi(x)$ is a solution then so is $\psi\left(x+C_{1} \varepsilon\right)$ for any $C_{1}$ and since

$$
\psi\left(x+C_{1} \varepsilon\right) \cong \psi(x)+C_{1} \varepsilon \psi^{\prime}(x)+O\left(\varepsilon^{2}\right),
$$

$C_{1}$ must be arbitrary.

3. Nonexistence of solutions. In the preceding section we have demonstrated for type I (type II) superconductors that superconducting solutions may exist only for $\kappa<$ $h<1 / \sqrt{2}(1 / \sqrt{2}<h<\kappa)$. However, this result was derived only for $\kappa$ values such that $|\kappa-1 / \sqrt{2}| \ll 1$. In the following we wish to demonstrate that the validity of this result may be extended to arbitrary values of $\kappa$. More explicitly, our goal would be to prove the following claim.

THEOREM 1. Suppose that $\kappa<1 / \sqrt{2}(\kappa>1 / \sqrt{2})$ and either

a. $h>1 / \sqrt{2}(0<h<1 / \sqrt{2})$

or

b. $0<h<\kappa(h>\kappa)$.

Then no solution of (1.1), for which $0<\psi$ for all $x$, can exist.

The case $h=0$ has been excluded, since $\psi \equiv 1, A \equiv 0$ is then a solution.

Our first step would be to state the following lemma [8].

LEMMA 1. Any solution of (1.1) has the following properties:
a. $A^{\prime}>0 \forall x$,
b. $-1<\psi<1 \forall x$.
c. Suppose that for some $x_{0}$ we have: $\psi\left(x_{0}\right)>0, \psi^{\prime}\left(x_{0}\right) \leq 0$. Then,

$$
\psi(x)>0 \quad \forall x>x_{0} \quad \Rightarrow \quad \psi^{\prime}(x)<0 \quad \forall x>x_{0} .
$$

d. $\psi<e^{-K x^{2}}$ for some positive $K$, and hence $A^{\prime} \sim h+O\left(e^{-K x^{2}}\right)$ as $|x| \rightarrow \infty$.

Note that the arguments invoked by Chapman et al. [8] to show that $\psi$ must be positive are not valid in our case because the boundary conditions at $-\infty$ in the problem they study are different than ours. Furthermore, $\psi$ is not necessarily positive in our case. The results of linear bifurcation theory [5] clearly indicate that solutions, for which $\psi<0$ on some subsets of the real line, must exist. However, since solutions for which $\psi$ is negative are not of any practical interest (and since they were found unstable by linear stability analysis [6]), we shall confine the discussion to the "physical" solutions for which $\psi$ is nonnegative.

Our next step would be to prove the following symmetry property. 
LEMma 2. Any solution of (1.1) for which $\psi>0 \forall x$ is symmetric, i.e., $\psi^{\prime}(0)=0 \Leftrightarrow$ $A(0)=0$.

Note that the choice $x=0$ is arbitrary in view of the translational invariance of (1.1).

Proof. Suppose for a contradiction that there exists a solution $\left(\psi_{1}, A_{1}\right)$ of $(1.1)$ for which $A_{1}(0)=0, \psi_{1}^{\prime}(0)<0$. Then, the pair $\left(\psi_{2}, A_{2}\right)$ defined by $\psi_{2}(x)=\psi_{1}(-x)$, $A_{2}(x)=-A_{1}(-x)$ is also a solution of (1.1) for which $\psi_{2}^{\prime}>0$.

Following [8] we define

$$
u=\left(A^{\prime}\right)^{2} ; \quad R=A^{2}
$$

and use the Hamiltonian relation

$$
\frac{\left(\psi^{\prime}\right)^{2}}{\kappa^{2}}+\left(A^{\prime}\right)^{2}-\frac{1}{2} \psi^{4}+\psi^{2}-\psi^{2} A^{2}=h^{2}
$$

together with the identities

$$
\begin{aligned}
\psi^{2} & =\frac{d u}{d R}, \\
\left(\psi^{\prime}\right)^{2} & =\frac{R u}{\frac{d u}{d R}}\left(\frac{d^{2} u}{d R^{2}}\right)^{2} \quad \text { for } R>0
\end{aligned}
$$

to obtain the equation

$$
\frac{R}{\kappa^{2}}\left(\frac{d^{2} u}{d R^{2}}\right)^{2}+\frac{d u}{d R}=\frac{1}{u}\left\{\frac{1}{2}\left(\frac{d u}{d R}\right)^{2}-\frac{d u}{d R}+R \frac{d u}{d R}+h^{2}\right\} \frac{d u}{d R} .
$$

Define $u_{1}=\left(A_{1}^{\prime}\right)^{2}, u_{2}=\left(A_{2}^{\prime}\right)^{2}$; hence, $\psi_{1}^{2}=\frac{d u_{1}}{d R}, \psi_{2}^{2}=\frac{d u_{2}}{d R}$. Obviously $u_{1}(0)=u_{2}(0)$, $\frac{d u_{1}}{d R}(0)=\frac{d u_{2}}{d R}(0)$, and $u_{1}(\infty)=u_{2}(\infty)$. However, since $\psi_{1}^{\prime}(0)<0$ and $\psi_{2}^{\prime}(0)>0, u_{1}$ and $u_{2}$ satisfy initially different equations. Explicitly, $u_{1}$ satisfies the equation

$$
\frac{R^{1 / 2}}{\kappa} \frac{d^{2} u_{1}}{d R^{2}}=-\left\{\frac{d u_{1}}{d R}\left(\frac{1}{u_{1}}\left[\frac{1}{2}\left(\frac{d u_{1}}{d R}\right)^{2}-\frac{d u_{1}}{d R}+R \frac{d u_{1}}{d R}+h^{2}\right]-1\right)\right\}^{1 / 2}
$$

whereas $u_{2}$ satisfies

$$
\frac{R^{1 / 2}}{\kappa} \frac{d^{2} u_{2}}{d R^{2}}=\left\{\frac{d u_{2}}{d R}\left(\frac{1}{u_{2}}\left[\frac{1}{2}\left(\frac{d u_{2}}{d R}\right)^{2}-\frac{d u_{2}}{d R}+R \frac{d u_{2}}{d R}+h^{2}\right]-1\right)\right\}^{1 / 2} .
$$

While (3.2) is valid for any positive value of $R,(3.3)$ remains valid as long as $\psi_{2}^{\prime}>0$. In view of Lemma $1 \mathrm{~d}$, at some $R_{0}>0$ we must have $\frac{d^{2} u_{2}}{d R^{2}}=0$. Setting $\Phi=u_{2}-u_{1}$, for $0<R<R_{0}$ we have $\frac{d^{2} \Phi}{d R^{2}}>0$; hence, since $\Phi(0)=\frac{d \Phi}{d R}=0$ both $\Phi\left(R_{0}\right)$ and $\frac{d \Phi}{d R}\left(R_{0}\right)$ must be positive.

Following [8] once again, we write the equation for $\Phi$, for $R>R_{0}$, in the following form:

$$
\frac{R}{\kappa^{2}}\left(\frac{d^{2} u_{1}}{d R^{2}}+\frac{d^{2} u_{2}}{d R^{2}}\right) \frac{d^{2} \Phi}{d R^{2}}+(\cdots) \frac{d \Phi}{d R}+\frac{1}{u_{1} u_{2}}\left[\frac{1}{2}\left(\frac{d u_{2}}{d R}\right)^{2}-\frac{d u_{2}}{d R}+R \frac{d u_{2}}{d R}+h_{2}\right] \frac{d u_{2}}{d R} \Phi=0
$$


The precise form of the coefficient $(\cdots)$ is irrelevant here, since, recalling that

$$
\begin{gathered}
\frac{d^{2} u_{1}}{d R^{2}}+\frac{d^{2} u_{2}}{d R^{2}}<0 \text { for } R>0, \\
\frac{1}{u_{2}}\left[\frac{1}{2}\left(\frac{d u_{2}}{d R}\right)^{2}-\frac{d u_{2}}{d R}+R \frac{d u_{2}}{d R}+h_{2}\right] \frac{d u_{2}}{d R}=\frac{R}{\kappa^{2}}\left(\frac{d^{2} u_{2}}{d R^{2}}\right)^{2}+\frac{d u_{2}}{d R}>0
\end{gathered}
$$

we see that $\Phi$ cannot have a positive maximum for $R>R_{0}$, which clearly contradicts $\Phi(\infty)=0$.

It is now clear that $A(0)=0 \Rightarrow \psi^{\prime}=0$. Suppose now that $\psi^{\prime}=0$ but $A(0)<0$. By Lemma 1a, at some $x_{0}, A$ must vanish. By Lemma 1c, $\psi^{\prime}\left(x_{0}\right)$ must be negative, which is impossible as we have just proved. If $A(0)>0$ and $\psi^{\prime}=0$ we apply the transformation

$$
\psi(x) \rightarrow \psi(-x) ; \quad A(x) \rightarrow-A(-x)
$$

and proceed as before.

It is interesting to note here that when studying (1.1) on a finite interval, nonsymmetric solutions can exist [3], [4] though the problem is symmetric. The arguments applied in the proof of Lemma 2 are not valid on finite intervals since the problem is not translation invariant there.

Lemma 2 allows us to prove the following result.

LEMma 3. Consider the function

$$
G=\frac{\psi^{\prime}}{\kappa}+\psi A
$$

For type I (type II) superconductors, $G$ must be nonpositive (nonnegative) $\forall x \geq 0$.

Proof. It is easy to show that $G$ satisfies the relation

$$
G^{\prime \prime}-\left[\kappa A+\frac{\psi^{\prime}}{\psi}\right] G^{\prime}-\left[2 \kappa^{2} \psi^{2}-\kappa A \frac{\psi^{\prime}}{\psi}+\kappa A^{\prime}\right] G=\left(1-2 \kappa^{2}\right) \psi^{3} A
$$

Obviously for type I superconductors, $G$ cannot have a positive maximum, whereas for type II it cannot have a negative minimum. The lemma is now proved since by Lemma $2, G(0)=0$ and by Lemma $1 \mathrm{~d}, G(\infty)=0$.

An immediate consequence of Lemma 2 is that when $\kappa=1 / \sqrt{2}, G$ must identically vanish. Hence every solution of (1.1) must be a solution of (1.3).

Proof of Theorem 1. We shall consider only type I superconductors. The proof for type II superconductors is almost identical. We first prove part (a) of the theorem.

Suppose for a contradiction that although $h>1 / \sqrt{2}$, a solution $(\psi, A)$ exists for $(1.1)$. Following [7] we define the function

$$
F=\psi^{2}-1+\sqrt{2} A^{\prime}
$$

By Lemma 1 we have

$$
G^{\prime}>\kappa(\psi F+A G)
$$

The Hamiltonian relation (3.1) may be represented in terms of the functions $F$ and $G$ in the form

$$
F\left(\psi^{2}-1-\sqrt{2} A^{\prime}\right)-2 G\left(\frac{\psi^{\prime}}{\kappa}-\psi A\right)=-2\left(h^{2}-\frac{1}{2}\right) .
$$


Since $G(0)=0, \psi<1$, and $A^{\prime}>0$ we must have $F(0)>0$, and hence, by $(3.4), G^{\prime}(0)>0$, which clearly contradicts Lemma 3 .

We now prove part (b) by invoking informal arguments. We first note that by Lemma $1 \mathrm{~d}$, when $x \rightarrow \infty, \psi$ is approximately, up to an exponentially small error, a solution of

$$
\psi^{\prime \prime}-\kappa^{2}\left[(h x+a)^{2}-1\right] \psi=0
$$

in which $a=\lim _{x \rightarrow \infty}(A-h x)$. We seek an asymptotic approximation for the solution of (3.5) as $x \rightarrow \infty$. To this end we set the WKB expansion [13]

$$
\psi=\exp \left\{w_{1}+w_{2}+w_{3}+\cdots\right\}
$$

wherein $w_{n} \sim o\left(w_{n-1}\right)$. Substitution into (3.5) yields

$$
w_{1}=\frac{1}{2} \kappa h x^{2}, \quad w_{2}=-\kappa a x, \quad w_{3}=-\frac{h-\kappa}{h} \log x
$$

from which we obtain

$$
G=\left(-\frac{h-\kappa}{\kappa h x}+O\left(x^{-2}\right)\right) \psi .
$$

Obviously $h$ must be greater than $\kappa$, otherwise Lemma 3 is violated once again.

We note that in both cases, for $h>1 / \sqrt{2}$ and for $h<\kappa$ (for type I), Lemma 3 is violated. However, in the first case the negativity of $G$ is violated near $x=0$, whereas in the latter one it is violated as $x \rightarrow \infty$.

4. Concluding remarks. In the following we comment on the significance of the previous results to time-dependent analysis.

The linear stability of the solutions to (1.1) has been studied by Chapman [6] in the case $\psi \ll 1$. His results clearly show that superconducting solutions are stable for type II superconductors and unstable for type I. Expecting that these results would be still valid for solutions for which $\psi$ is not necessarily small, it seems plausible that for type I superconductors, the solutions obtained in Sec. 2 are not of any practical importance since they are unstable. On the other hand, we expect to have stable solutions for type II superconductors when $1 / \sqrt{2}<h<\kappa$. Our energy calculations support this assumption: the superconducting solutions were found to have lower (higher) energy than the normal state for type II (type I) superconductors. The results of Sec. 3 clearly show that such "mixed state" solutions do not exist when either $h<1 / \sqrt{2}$ or $h>\kappa$. Linear stability analysis [6] shows that for $h>\kappa$ the normal solution (1.2) is stable; however, for $0<h<1 / \sqrt{2}$ no stable solution of (1.1) exists. In the latter case, the long-time solution of the time-dependent Ginzburg-Landau equation cannot be steady.

If we look at the leading-order solution of $(2.2), \psi_{0}$ we see that as $h_{1} \rightarrow 0, \bar{\psi} \rightarrow$ 1. Looking at Chapman's [5] plots of $\psi_{0}$ we see that as $\bar{\psi}$ becomes closer to 1 the domain in which $\psi_{0}$ is very close to 1 becomes larger. This domain constitutes a purely superconducting region around $x=0$. In addition, two transition layers and two normal regions, as $x \rightarrow \pm \infty$, exist. The solution inside the purely superconducting region is (almost) $\psi=1, A=0$. In the normal region it is approximately (1.2) and the transitions 
layer, formed around $x= \pm x_{0}\left(h_{1}\right)$ (at which $\psi=1 / 2$ say), approximately satisfies the following boundary conditions:

$$
\begin{gathered}
\psi \rightarrow 1, A \rightarrow 0 \quad \text { as } \quad \operatorname{sign}\left(x_{0}\right) x-x_{0} \rightarrow-\infty, \\
\psi^{\prime} \rightarrow 1, A^{\prime} \rightarrow \frac{1}{\sqrt{2}} \quad \text { as } \quad \operatorname{sign}\left(x_{0}\right) x-x_{0} \rightarrow \infty .
\end{gathered}
$$

The qualitative features of the solution of (1.1a) and (1.1b) together with the above boundary conditions, have been thoroughly discussed in [8]. For type II superconductors when $h>1 / \sqrt{2}$ we expect that the transition layers would save as wavefronts propagating towards $\pm \infty$. The velocity of propagation should be determined by the free-boundary model obtained by Chapman [7].

For large but finite intervals we expect that the solution would reach a steady state when the wave fronts hit the edges. Yet, the solution is expected to be of a completely different nature than the solutions obtained in this work or from any linearized solution [12]. It is more likely to be wholly superconducting in the bulk of the material, whereas near the edges a boundary layer is expected. Such a solution was provided in [10].

Acknowledgments. Dr. S. J. Chapman is acknowledged for suggesting to the author the idea of performing the asymptotic analysis in Sec. 2. The author wishes to thank Professor J. Rubinstein for a lot of helpful comments.

\section{REFERENCES}

[1] M. S. Berger, Creation and breaking of self-duality symmetry-a modern aspect of calculus of variations, Contemp. Math. 17, Amer. Math. Soc., Providence, RI, 1983, pp. 379-394

[2] E. Bogomolnyi, The stability of classical solutions, Sov. Jour. Nucl. Phys. 24, 449-454 (1976)

[3] C. Bolley and B. Helffer, Rigorous results on Ginzburg-Landau models in a film submitted to an exterior parallel magnetic field I, Nonlinear Stud. 3, 1-29 (1996)

[4] C. Bolley and B. Helffer, Rigorous results on Ginzburg-Landau models in a film submitted to an exterior parallel magnetic field II, Nonlinear Stud. 3, 121-152 (1996)

[5] S. J. Chapman, Nucleation of superconductivity in decreasing fields I, EJAM 5, 449-468 (1994)

[6] S. J. Chapman, Nucleation of superconductivity in decreasing fields II, EJAM 5, 469-494 (1994)

[7] S. J. Chapman, Asymptotic analysis of the Ginzburg-Landau model of superconductivity: reduction to a free boundary model, Quart. Appl. Math. 53, 601-627 (1995)

[8] S. J. Chapman, S. D. Howison, J. B. Mcleod, and J. R. Ockendon, Normal/Superconducting transition in Landau-Ginzburg Theory, Proc. Roy. Soc. Edinburgh 119A, 117-124 (1991)

[9] A. T. Dorsey, Dynamics of interfaces in superconductors, Ann. Phys. 233, 248-269 (1994)

[10] V. A. Ginzburg and L. D. Landau, On the theory of superconductivity, Soviet Phys. JETP 20, 1064-1082 (1950)

[11] J. Kevorkian and J. D. Cole, Perturbation Methods in Applied Mathematics, Applied Mathematical Sciences, vol. 34, Springer-Verlag, New York, 1981

[12] M. H. Millman and J. B. Keller, Perturbation theory of nonlinear boundary value problem, J. Math. Phys. 10, 342-361 (1969)

[13] J. D. Murray, Asymptotic Analysis, second edition, Applied Mathematical Sciences, vol. 48, Springer-Verlag, New York, 1984 